\title{
A fast feature extraction algorithm for image and video processing
}

\begin{abstract}
Medical images and videos are utilized to discover, diagnose and treat diseases. Managing, storing, and retrieving stored images effectively are considered important topics. The rapid growth of multimedia data, including medical images and videos, has caused a swift rise in data transmission volume and repository size. Multimedia data contains useful information; however, it consumes an enormous storage space. Therefore, high processing time for that sheer volume of data will be required. Image and video applications demand for reduction in computational cost (processing time) when extracting features. This paper introduces a novel method to compute transform coefficients (features) from images or video frames. These features are used to represent the local visual content of images and video frames. We compared the proposed method with the traditional approach of feature extraction using a standard image technique. Furthermore, the proposed method is employed for shot boundary detection (SBD) applications to detect transitions in video frames. The standard TRECVID 2005, 2006, and 2007 video datasets are used to evaluate the performance of the SBD applications. The achieved results show that the proposed algorithm significantly reduces the computational cost in comparison to the traditional method.
\end{abstract}

Keyword: Block processing; Feature extraction; Orthogonal polynomials; Shot boundary detection; Temporal video segmentation; Abrupt transition detection 